\title{
Konfliktminerale und Lieferkettenmanagement mineralischer Rohstoffe
}

\author{
Felix Gerald Giller ${ }^{1,2}$ und Michael Tost ${ }^{1}$
}

${ }^{1}$ Lehrstuhl für Bergbaukunde, Bergtechnik und Bergwirtschaft, Department Mineral Resources Engineering, Montanuniversität Leoben, Leoben, Österreich ${ }^{2}$ Wien, Österreich

Eingegangen 26. März 2019; angenommen 24. April 2019; online publiziert 15. Mai 2019

Zusammenfassung: Bewaffnete rebellische Gruppierungen in der Demokratischen Republik Kongo und den Nachbarstaaten werden meist durch das illegale Fördern und Verkaufen von Mineralen, welche die Elemente Zinn, Wolfram, Tantal und Gold enthalten, finanziert. Durch Regulierungsinitiativen sollen diese Gruppierungen finanziell ausgetrocknet werden.

Diese Arbeit gibt einen Überblick über die gängigen Initiativen, insbesondere der US und der EU-Regulierung und zeigt deren Schwierigkeiten auf.

In der Demokratischen Republik Kongo hängt die wirtschaftliche Grundlage von circa 10 Mio. Menschen vom artisanalen Bergbau ab. Aus diesem Grund wird die Möglichkeit untersucht, eine transparente und vertrauenswürdige Lieferkette zu erstellen, um weiter Minerale aus den betroffenen Regionen zu beziehen, ohne bewaffnete Konflikte zu finanzieren.

Schlüsselwörter: Konfliktminerale,

Lieferkettenmanagement, Blockchain, Demokratische Republik Kongo

Conflict Minerals and Supply Chain Management of Mineral Resources

Abstract: Armed rebellious groups in the Democratic Republic of Congo and neighbouring states are mostly financed by the illegal mining and sale of minerals containing the elements tin, tungsten, tantalum and gold. Through regulatory initiatives, these groups are to be dried up financially.

This paper gives an overview of the current initiatives, in particular the US and EU regulation, and highlights their difficulties.

F. G. Giller (ه)

Alxingergasse $31 / 5$

1100 Wien, Österreich

felix.gerald.giller@gmail.com
In the Democratic Republic of Congo, the economic base of approximately 10 million people depends on artisanal mining. For this reason, the possibility of establishing a transparent and trustworthy supply chain to continue sourcing minerals from the affected regions without financing armed conflict is being explored.

Keywords: Conflict minerals, Supply chain management, Blockchain, Democratic Republic of Congo

\section{Einleitung}

Nach den Bürgerkriegen in der Demokratischen Republik Kongo und den angrenzenden Staaten wird der Osten des Landes von nichtstaatlichen bewaffneten Gruppierungen kontrolliert. In dieser Region werden unter menschenrechtverletzenden Bedingungen die Mineralien für die Herstellung von Zinn, Wolfram, Tantal und Gold gefördert. Mit dem Erlös werden Waffen gekauft und Konflikte finanziert, weshalb sie häufig als Konfliktmineralien bezeichnet werden.

Die Vereinigten Staaten von Amerika und die Europäische Union haben Gesetze beziehungsweise Verordnungen erlassen, welche den Import von Konfliktmineralien verbieten.

Ziel dieser Arbeit ist es, die aktuellen nationalen und internationalen Regelungen zu Konfliktmineralien darzustellen, zu interpretieren und deren Problematiken aufzuzeigen. In einem weiteren Schritt wird festgehalten, welche Möglichkeiten es gibt, eine transparente Lieferkette zu gestalten. Es handelt sich hierbei um eine Analyse der aktuellen rechtlichen Lage und gängiger Lieferkettensysteme unter Zuhilfenahme von Gesetzestexten, Fachartikeln, Normen und weiterer Literatur. Unter Berücksichtigung der Blockchain-Technologie wird ein möglicher Anwendungsfall praktisch dargestellt. Es werden die Unterschiede und die Problematiken der aktuellen Regelungen angeführt. Die größte Schwierigkeit besteht darin, dass es sehr schwer und teuer geworden ist, legal gewonnene Minerale zu im- 
portieren, da der Importeur eine Berichterstattungspflicht zu tragen hat, um zu beweisen, dass es sich nicht um Konfliktminerale handelt. Es ist zu einem De-facto-Handelsembargo für Kongolesische Importe gekommen. Firmen haben den Anbieter gewechselt, um der Berichterstattungspflicht zu entgehen.

Eine transparente und vertrauenswürdige Lieferkette würde dazu beitragen, legale kongolesische Produkte wieder auf dem Weltmarkt zu handeln. Es werden gängige Methoden zum Aufbau einer transparenten Lieferkette vorgestellt. Auch die Blockchain wird untersucht, da mit diesem Transaktionssystem alle Beteiligten Zugriff auf aktuelle digitale Prozesse haben, diese aber nicht ändern können. Dies würde ein Höchstmaß an Sicherheit bieten.

\section{Konfliktminerale}

Unter dem Begriff Konfliktminerale versteht man jene Minerale, welche in Konfliktgebieten meist schnell und mit einfachen Mitteln abgebaut und anschließend zu hohen Preisen verkauft werden. Mit dem erzielten Gewinn werden Konflikte und (meist) nichtstaatliche bewaffnete Gruppierungen unterstützt [1]. Gesteinsverbände - mit einer hohen Konzentration an Wertmineralien - bergen ein erhebliches Entwicklungspotential, können allerdings in Konflikt- und Hochrisikogebieten den Anlass geben, die Staatsführung und Rechtsstaatlichkeit zu untergraben. In rohstoffreichen Konflikt- und Hochrisikogebieten sind Menschenrechtsverletzungen sehr weit verbreitet. Durch erwirtschaftete Erträge können rebellische Gruppierungen mehr Waffen erwerben und schwächen das staatliche System [2]. Als Konfliktminerale werden derzeit mineralische Rohstoffe bezeichnet, in welchen Zinn, Tantal, Wolfram, ihre Erze und Derivate sowie Gold enthalten sind. Sie werden sehr häufig für die Herstellung von Computern, Smartphones und anderen technischen Geräten verwendet und erlangen durch den rasch ansteigenden Konsum eine immer höhere Relevanz für die globalen Wirtschaftsströme. Es wird vermutet, dass zahlreiche Förderminen, vor allem in der Demokratischen Republik Kongo, aber auch in den Nachbarstaaten - Angola, Burundi, der Zentralafrikanischen Republik, der Republik Kongo, Kenia, Ruanda, Sudan, Tansania, Uganda und Sambia - von bewaffneten Gruppen kontrolliert werden [3]. Die Arbeit in einem solchen Bergbau erfolgt unter extremen Bedingungen und es ist sehr wahrscheinlich, dass Kinder und Zwangsarbeiter festgehalten werden. Häufig kommt es zu Unfällen, da die Arbeitssicherheit nicht gegeben ist. Die staatlichen, parastaatlichen oder sonstigen Machthaber der Regionen fordern willkürliche Abgaben oder Schutzgebühren von den Bergbaubetreibern und Händlern [4].

Im artisanalen Bergbau werden Mineralien mit einfachen Mitteln abgebaut. Er wird vorwiegend in Entwicklungs- und Schwellenländern betrieben [5]. Dieser Sektor hat sich aus der Not der Bevölkerung entwickelt und hat im Laufe der Zeit an Professionalität, wie beispielsweise durch die Einführung von Normen, dazugewonnen. Er wird in der DR Kongo vom Gesetz ausdrücklich anerkannt [6]. Weltweit hängt die Existenzgrundlage von 100 Mio. Menschen
- Arbeiter und ihre Familien - vom artisanalen Bergbau ab. Im Vergleich dazu steht der industrielle Bergbau, bei welchem circa $7 \mathrm{Mio}$. Menschen finanziell abgesichert sind [7]. In einem Bericht der Weltbank wurde geschätzt, dass die Existenzgrundlage von 8 bis 10 Mio. Menschen, welche in der DR Kongo leben, indirekt vom artisanalen Bergbau abhängt [8]. Diese Form des Kleinbergbaus ist auch ein Instrument zur Armutsbekämpfung und verhindert, dass noch mehr Menschen aus den ländlichen Regionen in die überfüllten Ballungsräume abwandern [5]

\section{Regulierungsinitiativen}

Um nichtstaatliche bewaffnete Gruppen nicht zu unterstützen und um zu verhindern, dass Gelder an illegale Organisationen fließen, haben sich einige Regulierungsinitiativen entwickelt. Diese sind teilweise auf regionaler und nationaler Ebene, allerdings gibt es auch eine ganze Reihe an internationalen Regelungen. Einige davon sind staatlich und scheinen als Gesetzestexte auf. Allerdings gibt es auch private Initiativen auf freiwilliger Basis, um die Minerale verantwortungsvoll zu beschaffen.

\subsection{Regionale und nationale Regulierungsinitia- tiven}

Auf eine Initiative des UN-Sicherheitsrates wurde im Jahr 2000 der wichtigste Rahmen für regionale und nationale Regelungen für zentralafrikanische Regionen gegründet, die "International Conference on the Great Lakes Region“ (ICGLR). Die Organisation hat folgende 12 Mitgliedsstaaten: Angola, Burundi, Zentralafrikanische Republik, Republik Kongo, Demokratische Republik Kongo, Kenia, Uganda, Ruanda, Süd Sudan, Sudan, Tansania, Sambia und wird von den Vereinten Nationen, der Afrikanischen Union, der Europäischen Union und zahlreichen Staaten unterstützt. Im Jahr 2006 wurde die "Regional Initiative against the illegal Exploration of Natural Resources" (RINR) gegründet, um die Finanzierung bewaffneter Konflikte durch Rohstoffeinnahmen zu unterbinden. Ein Teil der RINR befasst sich mit der Ausstellung von Zertifizierungen der Rohstoffe, Prüfung der Minen, der Nachvollziehbarkeit der Lieferkette in den Mitgliedsländern, der Erstellung einer Datenbank über Produktion, Handel und Export, sowie Kontrollen durch Dritte [9]. Die Schwierigkeit beziehungsweise Herausforderung besteht darin, die Vorgaben der strukturellen Veränderung der Rohstoffinitiativen in den Nationalstaaten umzusetzen [10].

\subsection{Dodd-Frank Act}

Im Jahr 2010 wurde vom US-Amerikanischen Kongress die Dodd-Frank Verordnung beschlossen. Sie wurde als Reaktion auf die Finanzmarktkrise von 2007 erlassen und hat die Förderung der Stabilität des US-Amerikanischen Finanzmarkts als Ziel. Ein Teil dieser Verordnung fordert den Ausschuss dazu auf, Vorschriften zu erlassen, welche Unter- 
nehmen, die als Hersteller gelten, dazu verpflichtet, ihre Verwendung von Konfliktmineralen offenzulegen. Die betroffenen Minerale sind, wie auch in den OECD-Leitsätzen, Wolfram, Tantal, Zinn und Gold. Diese Regelung betrifft Firmen, welche Konfliktminerale für die Herstellung oder die Produktion eines Produkts benötigen [11].

Für Unternehmen ist es sehr schwierig, diese Richtlinien zu erfüllen, da zu viele Zulieferer bis zum Endprodukt in der Lieferkette involviert sind. Als Folge dieses Mehraufwandes werden unter Umständen die Produkte teurer Unternehmen haben auf Verstoß gegen die US-Verfassung geklagt und recht bekommen, woraufhin die Unternehmen die Kennzeichnungen für "DRC-Konfliktfrei“, „DRC-Konflikt unbestimmbar" und „nicht DRC-Konfliktfrei“ nicht mehr an den Produkten anbringen müssen. Sämtliche anderen Vorschriften behalten allerdings die Gültigkeit [12]

Da der Dodd-Frank Act einen produktbezogenen Ansatz der Offenlegung der Lieferkette verfolgt, ergeben sich daraus ein paar Probleme:

- Ausländische Rohstoff- und Grundstofflieferanten können durch die Gesetzgebung nicht gebunden werden und somit lässt sich die Wahrheit der getätigten Aussagen nur schwer überprüfen.

- Die Anzahl der zu verwaltenden Zertifikate ist bürokratisch sehr aufwendig und folglich mit hohen Kosten verbunden [13]. Eine Studie der Tulane Universität hat ergeben, dass die Kosten für die Umsetzung dieser Maßnahmen 7,93 Mrd. Dollar betragen [14].

- Als Konsequenz wird ein Unternehmen die Zertifizierungspflicht umgehen und bei der Beschaffung der Rohstoffe auf andere Länder ausweichen, was zur Folge hat, dass die im Kongo arbeitende Bevölkerung durch den industriellen Bergbau von der Weltwirtschaft ausgeschlossen wird [13].

Zusammenfassend kann man sagen, dass die Durchführungsbestimmungen bei der Sektion 1502 des Dodd-Frank Acts eher vage sind und er auch keine Sanktionen gegen Firmen, welche das Gesetz nicht einhalten, beinhaltet [14].

\subsection{OECD - Leitsätze und die Verordnung (EU) $2017 / 821$}

2011 verabschiedete die OECD die "Leitsätze für Sorgfaltspflichten für Lieferketten von mineralischen Rohstoffen aus konfliktbetroffenen und stark gefährdeten Gebieten" nach einem zweijährigen Stakeholder-Prozess. Es wurde eine gemeinsame staatliche Initiative gegründet, welche die Erfüllung der Sorgfaltspflichten zur Förderung verantwortlicher Lieferketten für Minerale aus Konflikt- und Hochrisikogebieten forciert. Ziel ist die Einhaltung der Menschenrechte durch die Unternehmen und die Verhinderung einer Konfliktverschärfung bei der Mineralbeschaffung. Des Weiteren sollen die Lieferketten für Minerale transparent gehalten werden, damit durch den Abbau und Handel der Minerale nicht Konflikte und Menschenrechtsverletzungen ausgelöst werden, sondern stattdessen die Länder vom Abbau profitieren [1]. In Konflikt- und Hochrisikogebieten haben Unter- nehmen, welche Abbau und Handel mit wertvollen Mineralen betreiben, die Möglichkeit, durch Schaffung von Arbeitsplätzen den Wohlstand und somit die Entwicklung vor Ort zu fördern. Allerdings können Unternehmen auch zu negativen Entwicklungen beitragen, wie beispielsweise Menschenrechtsverletzungen und Auseinandersetzungen. Mit diesen Leitsätzen werden Rahmenbedingungen für die Erfüllung der Sorgfaltspflicht geschaffen. Die Leitsätze beziehen sich ausschließlich auf die Einfuhr von Erzen und Konzentraten, sofern diese Wolfram, Zinn, Tantal oder Gold enthalten und bieten keine Einschränkung auf bestimmte Herkunftsländer. Es liegt also im Ermessen des EU-Unternehmens, welches die Minerale nach Europa importiert, ob ein Land als Konflikt- oder Hochrisikogebiet zu behandeln ist oder nicht. Ein weiterer Unterschied zum amerikanischen Gesetz ist auch, dass es sich ausschließlich auf Importeure von unverarbeiteten Rohstoffen und Hüttenprodukten bezieht und nicht auch auf Halbfertig- und Fertigprodukte, in welchen die Rohstoffe enthalten sind [1]. Die Leitsätze der OECD sehen vor, dass der EU-Importeur die Berichterstattungspflicht zu tragen hat und nicht wie im amerikanischen System, die Verkäufer des Endproduktes. Dadurch sind nur circa 400 Firmen in der Europäischen Union berichterstattungspflichtig [12].

Im Jahr 2017 wurde die EU-Verordnung 2017/821 zur verpflichtenden Einhaltung der OECD-Leitsätze erlassen.

\subsection{Auswirkungen der Regulierungsinitiativen}

Die angeführten Initiativen umfassen Schritte, wie die Verabschiedung eines Handbuchs zur Zurückverfolgbarkeit, Zertifizierungen für Rückverfolgungen und weitere Sorgfaltspflichten. Allerdings stellen einige der Maßnahmen auch ein De-facto-Handelsembargo für kongolesische Exporte dar, da Firmen teilweise den Zulieferer wechseln, um die Sorgfaltspflichten zu vermeiden [6]. Aufgrund der Tatsache, dass bis zu 10 Mio. Menschen in der DR Kongo ihren Lebensunterhalt direkt oder indirekt durch den artisanalen Bergbau verdienen, sollten weitere Schritte gesetzt werden, um der dort lebenden Bevölkerung das Einkommen zu sichern [15]. Initiativen wie der Dodd-Frank Act und die EU-Verordnung sind wichtige Anhaltspunkte, um den Konsumenten dazu zu bringen, Verantwortung zu übernehmen. Diese endet allerdings nicht ausschließlich bei der Wahrung der Menschenrechte. Andere Aspekte, wie der Umweltschutz oder der Arbeitnehmerschutz, müssen ebenfalls berücksichtigt werden [16].

\section{Lieferkettenmanagement und Blockchain}

Die beschriebenen Probleme einer Lieferkette sind bei Firmen, welche mit mineralischen Rohstoffen arbeiten, sehr häufig am Beginn zu finden. Die Herausforderung besteht darin, ein System zu finden, welches den Ansprüchen der Stakeholder gerecht wird, um Zwangsarbeit und Menschenrechtsverletzungen in einem Betrieb zu eliminieren. Ein weiterer wichtiger Aspekt einer transparenten Lieferkette ist die Umweltbelastung. Nicht nur in der Gewinnung, 
sondern auch bei Raffinationsprozessen entstehen schädliche Gase und Abwässer. Von diesem Problem sind nicht nur Entwicklungsländer betroffen, sondern auch Länder in der EU, sowie China und Amerika [17]. Es ist wichtig, die Ziele der nachhaltigen Lieferkette in Form von Vorschriften, Schulungen, Beratungen und Anreizen in die bestehenden Beschaffungsprozesse proaktiv zu integrieren. So können ethische, soziale und ökologische Mindestanforderungen an die Lieferanten vertraglich festgehalten werden und jene damit rechtswirksam zu deren Einhaltung verpflichtet werden [18]. Mithilfe der Blockchain-Technologie könnte eine überwachbare Lieferkette geschaffen werden. Die Blockchain ist ein dezentral gespeichertes Transaktionsprotokoll, welches jede Änderung transparent erfasst. Ihr größter Vorteil ist die Fähigkeit, Vertrauen zwischen zwei Transaktionspartnern zu schaffen, ohne dass sie von einer dritten Person vermittelt werden [19].

Eine transparente und vertrauenswürdige Lieferkette würde dazu beitragen, legale kongolesische Produkte wieder auf dem Weltmarkt zu handeln. Die Anwendung der Blockchain würde ein Höchstmaß an Sicherheit bieten.

Funding. Open access funding provided by Montanuniversität Leoben.

Open Access Dieser Artikel wird unter der Creative Commons Namensnennung 4.0 International Lizenz (http://creativecommons.org/licenses/ by/4.0/deed.de) veröffentlicht, welche die Nutzung, Vervielfältigung, Bearbeitung, Verbreitung und Wiedergabe in jeglichem Medium und Format erlaubt, sofern Sie den/die ursprünglichen Autor(en) und die Quelle ordnungsgemäß nennen, einen Link zur Creative Commons Lizenz beifügen und angeben, ob Änderungen vorgenommen wurden.

\section{Literatur}

1. Küblböck, K.: EU-Verordnung zu „Konfliktmineralien“ - ein Schritt zu höherer Rechenschaftspflicht im Rohstoffsektor, Wien: ÖFSE Österreichische Forschungsstiftung für Internationale Entwicklung, 2018

2. Verordnung (EU) 2017/821 des Europäischen Parlaments und des Rates vom 17. Mai 2017 zur Festlegung von Pflichten zur Erfüllung der Sorgfaltspflichten in der Lieferkette für Unionseinführer von Zinn, Tantal, Wolfram, deren Erzen und Gold aus Konflikt- und Hochrisikogebieten, http://data.europa.eu/eli/reg/2017/821/oj

3. Futurezone: https://futurezone.at/netzpolitik/eu-will-einfuhr-vonmineralien-aus-konfliktzonen-stoppen/252.361.193 (02.08.2018)

4. Baggehufwudt, U., BGR: https://www.bgr.bund.de/DE/Themen/ Zusammenarbeit/TechnZusammenarbeit/Laender/kongo_dr.html? $\mathrm{nn}=1542184$ (09.08.2018)

5. Wotruba, H.: Kleinbergbau und seine Umweltauswirkungen - von traditionellen Methoden zu angepaßter Technologie, Vortrag am
Geoökologischen Kolloquium WS 2004/05, Bayreuth: BayCEER, 2004

6. Geenen, S.; Marysse, S.: Democratic Republic of the Congo: Mining Sector, in: Tiess, G.; Majumder, T.; Cameron, P. (eds): Encyclopedia of Mineral and Energy Policy, Berlin, Heidelberg: Springer Link, 2016, pp. 1-6, https://doi.org/10.1007/978-3-642-40871-7

7. World Bank: http://www.worldbank.org/en/topic/extractiveindustries/ brief/artisanal-and-small-scale-mining, (22.08.2018)

8. World Bank: Democratic Republic of Congo. Growth with Goverance In the Mining Sector, Report No. 43402, 2008, https:// openknowledge.worldbank.org/handle/10986/8072

9. Küblböck, K.: Pinter, S.: Konfliktmineralien: Möglichkeiten und Grenzen aktueller Regulierungsinitiativen, Wien: ÖFSE Österreichische Forschungsstiftung für Internationale Entwicklung, 2016

10. Kraft, M.: Deutsche Gesellschaft für internationale Zusammenarbeit (GIZ), https://www.giz.de/de/weltweit/15662.html. (07.08.2018)

11. U. S. Securities and Exchange Commission: Fact sheet, Disclosing the use of conflict minerals, https://www.sec.gov/opa/Article/2012 2012-163htm---related-materials.html (06.08.2018)

12. Goldbacher, A.: Elektronik-Interview Konflikmineralien, Offenlegungspflichten entlang der Lieferkette,m https://www.elektroniknet. de/preview/-131906.html (07.08.2018)

13. John, K.: Konfliktrohstoffe, Positionspapier und Hintergrundpapier der Elektroindustrie, ZVEI - Zentralverband Elektrotechnik- und Elektronikindustrie e.V., Frankfurt am Main, 2013, https://www.zvei. org/presse-medien/publikationen/konfliktrohstoffe-positionspapierund-hintergrundpapier-der-elektroindustrie/ (24.04.2019)

14. Cuvelier, J.G.R.; Van Bockstael, S.; Vlassenroot, K.; Iguma, C.: Analyzing the impact of the Dodd-Frank Act on Congolese Livelihoods, https://s3.amazonaws.com/ssrc-cdn1/crmuploads/new_ publication_3/\%7B57858126-EF65-E411-9403-005056AB4B80\%7D. pdf (20 08 2018)

15. EurAc European Network for Central Africa: Accompanying Measures to the EU Regulation on Responsible Mineral Sourcing: towards an improved gonvernance of the artisanal mining sector in the DRC Position paper, Brussels, 2018. https://www.eurac-network.org/en

16. BME Bundesverband Materialwirtschaft, Einkauf und Logistik: Rohstoff-Lieferketten im Blick, https://www.bme.de/rohstoff-lieferketten im-blick-2215/ (28.09.2018)

17. Volkert, G.; Frank, K.-D.: Metallurgie der Ferrolegierungen, 2. Aufl., Berlin (u.a.): Springer, 1972

18. Müller, M.: Nachhaltige Lieferketten - Herausforderungen und Lösungsansätze, CSR-Reporting vor der Berichtspflicht, Berlin, 2015. Vortrag am 23.09.2016 in Berlin, Präsentationsunterlagen unter https://www.ranking-nachhaltigkeitsberichte.de/data/ranking/user_ upload/2015/Pr\%C3\%A4sentationen/M\%C3\%BCller_Universitaet_ UIm_Nachhaltige_Lieferketten-Herausforderungen_und Loesungsans\%C3\%A4tze.pdf

19. Bogart, S.; Rice, K.: The Blockchain Report: Welcome to the Internet of Value, Needham \& Company, 2015, S. 2-4

Hinweis des Verlags. Der Verlag bleibt in Hinblick auf geografische Zuordnungen und Gebietsbezeichnungen in veröffentlichten Karten und Institutsadressen neutral. 